\title{
FLOW FIELD MEASUREMENT OF MIXING DRIVEN BY BUOYANCY
}

\author{
W.M.B. Duval ${ }^{*}$, C. Batur ${ }^{+}$, H. Zhong ${ }^{+}$ \\ NASA Glenn Research Center, Cleveland, Ohio 44135* \\ University of Akron, Dept. of Mech. Eng., Akron, Ohio $44325^{+}$
}

\begin{abstract}
Mixing driven by buoyancy-induced flows inside a cavity consists of stretching and folding of an interface. Measurement of the flow field using particle imaging velocimetry shows that during stretching the flow field has a single elliptic point, thus dominated by a single vortex. However, global bifurcation that results in folding introduces a hyperbolic point whereby the flow field degenerates to multiple vortex interactions. The short-lived coherent structure observed during mixing which results in the RayleighTaylor morphology is attributed to vortex interactions.
\end{abstract}

\section{Introduction}

The mixing characteristics of non-homogeneous fluids driven by buoyancy are important towards understanding transport phenomenon in a microgravity environment. Mixing consists of stretching and folding of an interface due to a flow field whose intensity depends on the body force. For miscible liquids, the characteristic of the flow field determines whether mass transport is governed by diffusion or bulk stirring which induces mixing. For technologically important processes, transport of mass is governed by the coupling of the body force to scalar gradients such as concentration and or temperature $e^{1,2,3}$. In order to lend insight into these classes of problems we consider a model experimental system to study mixing driven by buoyancy-induced flows. The characteristics of mixing is addressed from detail measurements of the flow field using particle imaging velocimetry (PIV), and its corresponding interface dynamics using image processing techniques.

Two-dimensional flow field measurement using PIV has become a common method $^{4}$ to quantify instantaneous velocity fields. PIV yields the Eulerian velocity field. Its advantage over alternative methods such as Stereo Imaging Velocimetry which obtains the Lagrangian history of a limited number or particles, is that it allows the measurement of complex flow patterns. The basic mono 2-D PIV system can be extended to stereo configuration to measure the 3-D flow field. Alternate methods to measure 3-D flows include stereoscopic tracking velocimetry ${ }^{5}$. In addition to flow field information, differential quantities can also be estimated, for example 3-D applications of PIV have been used to measure the velocity gradient tensor field for turbulent flows ${ }^{6}$. Measurement of complex fields occurring during fingering displacement of a miscible interface ${ }^{7}$ has shown PIV to be a viable method for microgravity ${ }^{1}$ experiments. The PIV technique allows detail measurement of transient flow fields which is an important application towards our model problem.

We use PIV to measure the transient characteristics of flow fields due to buoyancy-induced mixing. The Lagrangian history of the interface motion is also measured in order to corroborate the length stretch history of the interface with the flow field. For certain parametric region the intensity of the flow field causes the interface to stretch and fold. Folding of the interface generates an internal breakwave which has a whorl-like structure similar to the Rayleigh-Taylor morphology. The global bifurcation of the flow field shows that stretching is due to an elliptic point in the flow field. This event is caused by an overturning motion of the flow field. The elliptic point bifurcates to a hyperbolic point during folding which gives rise to an internal breakwave. Catastrophic annihilation of the breakwave follows due to wall collision. The collision event causes sloshing oscillation of the two fluids which decays rapidly to a stably stratified configuration. Details of the flow field show that the oscillation causes transient vortices which serve as a stirring mechanism to induce mass transport.

In the following we show the experimental set-up to measure the flow field and to quantify the interface

Copyright @2003 by the American Institute of Aeronautics, Inc. The U.S. Government has a royalty-free license to exercise all rights under the copyright claimed herein for government purposes. All other rights by the Copyright owner. 